\title{
Fabrication of a Current-Perpendicular to Plane Magneto-resistive Device with a Dry Ice Blasting Lift-off Process
}

\author{
Kousaku Miyake, Yoshimi Hisatsune*, Masakiyo Tsunoda and Masashi Sahashi \\ Department of Electronic Engineering, Tohoku University, 6-6-05 Aramaki Aza Aoba, Aoba-ku, Sendai, 980-8579, Japan \\ *Department of Materials Science and Engineering, Kyushu University, 744 Motooka Nishi-ku, Fukuoka, 891-0395, Japan
}

Sub-micron sized magnetic tunnel junctions were fabricated using a dry ice blasting lift-off process for the resist on the magnetic tunnel junction. The fabricated devices showed little difference in transport characteristics from devices made using a conventional chemical lift-off process in an ultrasonic bath sonicator. The distinguished feature of the new process was the achievement of a soft and pure dry ice jet from a well-cooled nozzle with a clear liquid $\mathrm{CO}_{2}$ flow in a narrow orifice. There was little contamination or damage on the surface of the sample wafer with the pure jet. The dry ice blasting effectively removed the resist for smaller pillar devices with planar sizes of $<250 \mathrm{~nm}$.

Key words: magnetic tunnel junction, current perpendicular to the film plane device, dry ice blasting, lift-off process

\section{Introduction}

The areal densities of magnetic recording and magnetic random access memory devices have increased, recently. The utilized magnetoresistive (MR) devices, such as metallic current-perpendicular to the film plane (CPP) MR or tunneling MR (TMR) elements, have small planar size $<100 \mathrm{~nm}$. Such nano-sized MR elements are required to clarify the underlying physics and fundamental properties in basic research in the field of spintronics. However, it is not easy to fabricate a nano-device element in small laboratories or research groups. Normally, MR devices are fabricated from multi-layered thin films by etching of the films with a small-patterned resist mask, subsequent coating with insulator film, and finally opening to the outer electrodes on the top of the patterned films with a lift-off process. The lift-off process in an ultra-sonic bath with a remover is stochastic and is not necessarily complete even after a long time. Therefore, it is hard to define the process conditions.

Dry ice blasting is a unique method as a surface cleaner in semi-conductor manufacturing ${ }^{1,2)}$. The cleaning system basically consists of nozzle with narrow orifice which nozzle is connected with high pressurized $\mathrm{CO}_{2}$ (gas or liquid) cylinder. The accelerated dry ice particles mechanically and chemically remove dusts, burrs and surface organic contaminations. Therefore the dry ice blasting is advantageous for fabrication process of lift-off because the process difficult to remove a burrs on the device edges. Therefore the equipment has been utilized for Micro Electro Mechanical Systems (MEMS) and recording head devices fabrications. For electronics device fabrication, the equipment includes some attachments such as nozzle controller, anti-static electricity device, air chamber and temperature control stage as shown in Fig. 1(a). The size of the equipment is about $1 \times 2 \mathrm{~m}^{2}$ in footprint and $2 \mathrm{~m}$ in height. Dry ice blasting is similar to sand blasting but no solid residue on sample after the process at room temperature.
Dry ice particles, that are typically $<1 \mu \mathrm{m}$ in diameters ${ }^{3,4)}$ are generated when $\mathrm{CO}_{2}$ gas flows through a narrow orifice adiabatically. The dry ice jet might remove or damage soft resist on the surface of pillar devices. Besides, liquid $\mathrm{CO}_{2}$, generated from the dry ice cluster striking the surface, solves the surface organic contamination $^{2)}$ such as resist material. This lift-off process is useful, but damage to fabricated devices may occur, such as scratching, electrostatic discharge damage, and corrosion due to condensation.

In this article, small magnetic tunnel junctions (MTJs) with $\mathrm{MgO}$ barriers were fabricated using dry ice blasting for the lift-off process. The transport properties of the fabricated devices were evaluated to check for damage during the process.

\section{Experiment}

The sample film is a synthetic spin valve stacked structure consisting of thermally oxidized $\mathrm{Si}$ $\begin{array}{lllll}\text { substrate/Ta } & (5) / \mathrm{Ru} & (40) / \mathrm{Ta} & (5) / \mathrm{CoFeB} & (3) / \mathrm{MgO}\end{array}$ (0.8)/CoFeB (2.5)/CoFe (0.5)/Ru (0.85)/CoFe (2.5)/MnIr (7.5)/Ta (3)/Ru (10). Parentheses indicate the respective layer thickness in $\mathrm{nm}$.

In the first step, the film was etched into the bottom electrode pattern by photo-lithography and $\mathrm{Ar}$ ion milling. A small resist mask pattern was then formed by electron beam lithography using a TOK-TGMR negative resist (350 $\mathrm{nm}$ thickness) on the bottom electrode. A MTJ remained under the resist mask in successive $\mathrm{Ar}$ ion milling down to the bottom $\mathrm{CoFeB}$ layer. After the pillar patterning, the MTJ remaining resist mask was coated with $\mathrm{SiO}_{2}(35 \mathrm{~nm}) / \mathrm{Cu}(6 \mathrm{~nm})$ bilayer film as an insulating layer by magnetron sputtering. The surface $\mathrm{Cu}$ layer is deposited to provide an anti-static electricity during dry ice blasting. The sample wafer was then exposed to the dry ice blasting (sample A). A reference sample made without the dry ice blasting process was also fabricated (sample B). The MTJ in sample B was not coated with the additional $\mathrm{Cu}$ $(6 \mathrm{~nm})$ layer. Both sample wafers were dipped in 
remover, as in the normal lift-off process in an ultrasonic bath. After the lift-off process, the wafer surfaces were cleaned once by soft Ar milling in-situ to ensure a metallic contact, and then the top electrode was formed by $\mathrm{Cu}$ layer deposition $(200 \mathrm{~nm})$, followed by photo-lithography and $\mathrm{Ar}$ ion milling. Metallic $\mathrm{Cu}$ pillar device elements were also fabricated for evaluation of corrosion. Zenkyo \& $\mathrm{EPOCH} \mathrm{CO}_{2}$ cleaner equipment ${ }^{5)}$ was used in the dry ice blasting process.

\section{Dry ice blasting method}

A schematic illustration and a photograph of the work space of the dry ice blasting equipment are shown in Fig. 1. We chose an orifice of $0.15 \mathrm{~mm}$ diameter for the soft damage process. This diameter is relatively narrow compared to the typical diameter of $0.5 \mathrm{~mm}$. The nozzle size had an internal diameter of $1.5 \mathrm{~mm}$. The nozzle angle was 25 degrees from the horizontal film plane. The distance between the sample and the nozzle end was about $15 \mathrm{~mm}$. The raster scan step size of nozzle movement was $0.1 \mathrm{~mm} /$ line. The same blasting processes were carried out from 4 directions at intervals of 90 degrees by rotating the sample in the film plane. The atmosphere in the work space was filled with dry air, which was considered to be effective for ionization compared to conventional dry nitrogen. The dew point temperature in the work space was kept below $-25{ }^{\circ} \mathrm{C}$. The sample stage was kept at $40{ }^{\circ} \mathrm{C}$ during the blasting. The sample was protected from static electricity using a soft X-ray ionizer (L12645 Hamamatsu photonics Co. Ltd.) and grounded in the sample holder. The resistance between the sample surface and the ground was about $50 \Omega$.

Small dry ice particle is desirable for small MTJ fabrication without serious damage to the device. The dry ice particle is generated in a well-cooled nozzle with a clear flow of liquid $\mathrm{CO}_{2}$. A soft dry ice jet, which probably consists of pure and small dry ice particles, is generated from a narrow orifice with smaller particle velocity ${ }^{4}$. However, the narrow orifice does not allow flow of a large amount of liquid $\mathrm{CO}_{2}$, which results in insufficient cooling of the nozzle. The narrow orifice also tends to be blocked by contamination with frost. Therefore, a stable dry ice jet from a narrow orifice is hard to be achieved. As a result, the generation of the stable soft dry ice jet from narrow orifice has poor reproducibility in experiments. However, this difficulty can be resolved by changing the cooling method for the nozzle.

When liquid $\mathrm{CO}_{2}$ flows through the orifice and nozzle, the temperature of the nozzle gradually decreases. Water or organic material frost may cover the surface of nozzle with the decreasing of the nozzle temperature. In the first stage, a strong jet was observed, but the jet then became weak and unstable. Finally, the jet became strong and stable after 10 to 15 minutes after the start of $\mathrm{CO}_{2}$ flow. We suggest that the strong jet in the $1^{\text {st }}$ stage contains a large amount of $\mathrm{H}_{2} \mathrm{O}$ or organic particle in a high temperature nozzle because contamination spots appeared on the wafer surface during the $1^{\text {st }}$ stage. In contrast, with the stable strong jet was achieved in the final stage, the wafer surface had few contamination spots.

(a)

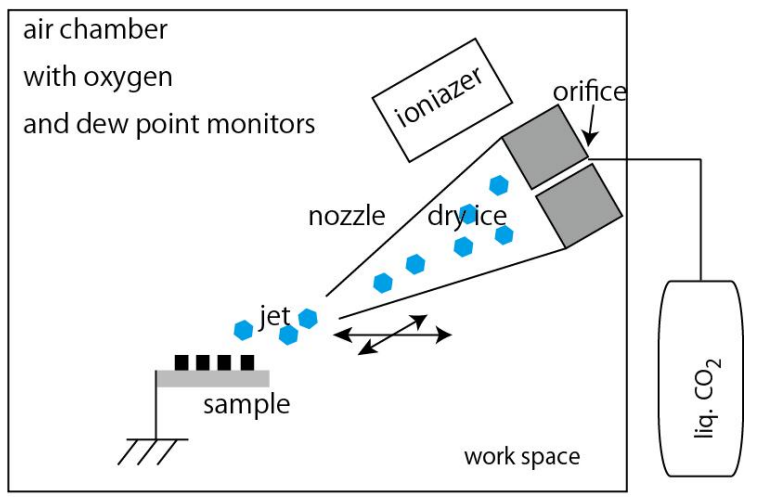

(b)

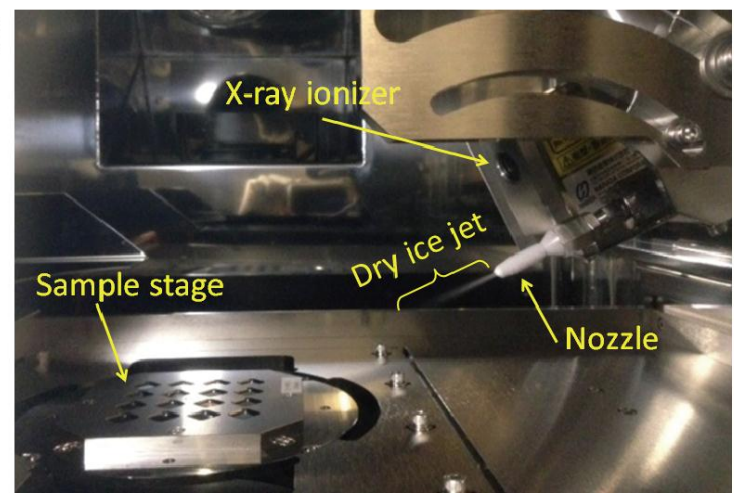

Fig. 1. (a) Schematic illustration of the dry ice blasting system in this experiment. (b) Photographic image of the actual equipment of the work space for the dry ice blasting apparatus. The $\mathrm{CO}_{2}$ nozzle is drawn back from the actual operating position to clearly show the dry ice jet. The $\mathrm{CO}_{2}$ nozzle was well frosted and a stable pure dry ice jet was obtained. The length of the dry ice jet in the photograph is approximately $30 \mathrm{~mm}$.

To achieve a clean process without contamination, a low temperature at the nozzle and a clear $\mathrm{CO}_{2}$ flow through the orifice were found to be important. However, the strength of the jet is not easy to judge from the appearance. The clear liquid $\mathrm{CO}_{2}$ flow in the nozzle can be monitored by the reduction in oxygen concentration in the work space. In the final stage, the monitored oxygen concentration was decreased linearly as shown in Fig. 2. A strong jet was achieved at the smallest concentration reduction of $-0.16 \% / \mathrm{min}$. (condition A) with thick frost on the nozzle (low temperature nozzle). However, the liquid $\mathrm{CO}_{2}$ flow was sometimes blocked. In this situation, the frost grew thin (high temperature nozzle) and the rate was $-0.13 \% / \mathrm{min}$. (condition B). Therefore, we can reproduce the jet condition by monitoring the reduction rate.

Atomic force microscopy images for metallic films $(\mathrm{Cu}$ and $\mathrm{FeCo}$ ) before and after blasting for the above 
processes with different jets (Fig. 3). The surfaces had little mechanical damages, such as holes and scratches independent of the material. However, fairly small contamination spots appeared on the surface with the condition $\mathrm{A}$ of $\mathrm{CO}_{2}$ jet, and large contamination spots appeared on the surface in the condition $\mathrm{B}$ of $\mathrm{CO}_{2}$ jet. Differences between the jets arise from the $\mathrm{CO}_{2}$ flow rate and resultant temperature of the nozzle. Therefore, a lower nozzle temperature is demanded for a clean process. The contamination material has not been identified. However, the colder nozzle in the condition A of $\mathrm{CO}_{2}$ jet was considered to be effectively absorbing $\mathrm{H}_{2} \mathrm{O}$ or organic molecules during the process as thick frost because the boiling point of these impurities generally greater than as that of pure $\mathrm{CO}_{2}$. Under these conditions, a pure $\mathrm{CO}_{2}$ jet was generated without contamination as a stronger and longer jet from the colder nozzle. Finally, we could make sure these good conditions and reproducibility by monitoring the oxygen concentration.

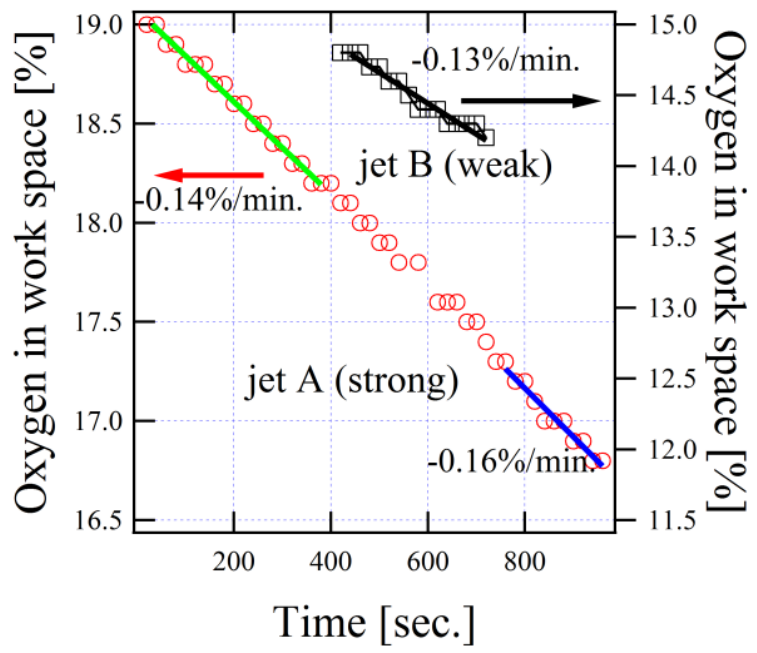

Fig. 2. Monitored oxygen concentration in work space during dry ice blasting process.

The resist on the MTJ in element fabrication was removed by the dry ice jet with the conditions $\mathrm{A}$ of $\mathrm{CO}_{2}$ jet. The condition of the jet was monitored by the rate of reduction of the oxygen concentration, thickness of frost, and the length of the jet determined visually.

\section{Experimental Results}

Scanning electron microscopy images (Fig. 4) were observed for devices of various sizes after the dry ice blasting process in sample $\mathrm{A}$. The resist and coated $\mathrm{SiO}_{2}$ film were almost removed by the blasting process only. As shown in Fig. 4, dry ice blasting entirely removed the resist and $\mathrm{SiO}_{2}$ film for smaller pillar-sized samples because the smaller resist has a light mass and is not mechanically hard. In contrast, in the larger devices of over $400 \mathrm{~nm}$ square, the resist and $\mathrm{SiO}_{2}$ film on the pillar could not be fully removed. Wall shaped $\mathrm{SiO}_{2}$ was left at the edge of the pillar in the larger devices (Fig. 4(d)). On the other hand, the lift-off process with conventional methods in sample $B$ required 1 hour for removal of the resist and $\mathrm{SiO}_{2}$ film from $58 \%$ devices and 2 hours for removal from $90 \%$ devices on a wafer.

Typical MR curves and the applied voltage dependence of resistance for devices of the same size are shown in Fig. 5. Similar characteristics were observed for samples A and B. Similar resistance area $(R A)$ products of $8.6 \pm 0.2$ and $9.1 \pm 0.7 \Omega \mu \mathrm{m}^{2}$ were obtained from the slope of the $R-1 / A$ plots for the large sized $(A>200 \times 200$ $\mathrm{nm}^{2}$ ) samples A and B, respectively (Fig. 6). Therefore, the MTJ had little damage from the blasting process. The endurance for the voltage stress (break-down voltage) was 0.5 to $1 \mathrm{~V}$ for typical samples. The unchanged transport properties in Fig. 5 indicate that the influence of static electricity during the dry ice blasting did not exceed the break down voltage.

Finally, a metallic $\mathrm{Cu}$ pillar device was also fabricated. The blasting process might cause corrosion of a metallic device. The obtained $R A$ of $2.23 \pm 0.07 \mathrm{~m} \Omega \mu \mathrm{m}^{2}$ from the slope of the $R-1 / A$ plots is sufficiently small for the 50 $\mathrm{nm}$ height of the $\mathrm{Cu}$ square pillar made from a sputtered film, and corresponds to $4.45 \mu \Omega \mathrm{cm}$ resistivity. This indicates that there was little corrosion of the $\mathrm{Cu}$ film during process.
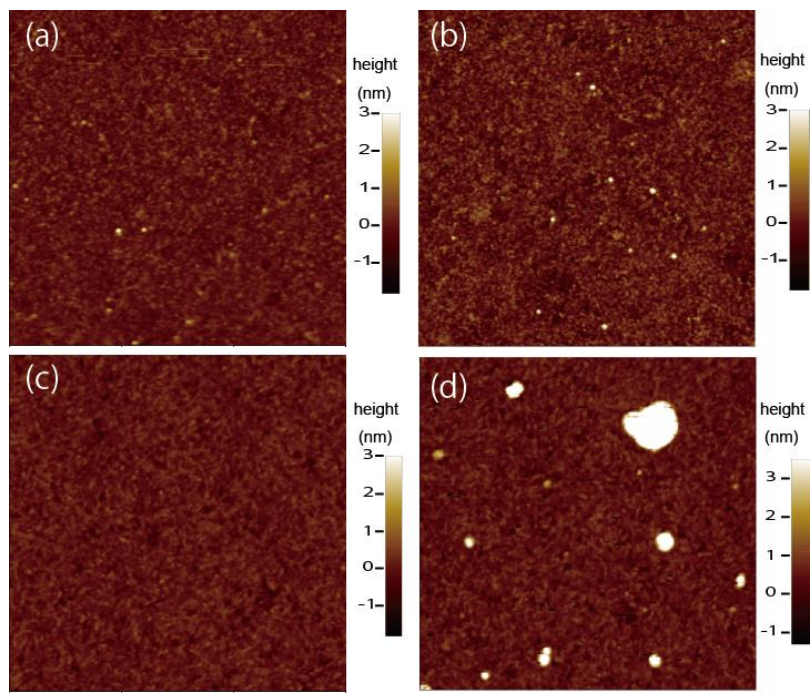

Fig. 3. Atomic force microscopy images $(\mathrm{a}, \mathrm{c})$ before and $(\mathrm{b}, \mathrm{d})$ after dry ice blasting of $(\mathrm{a}, \mathrm{b}) \mathrm{Cu}$ and (c,d) FeCo film surfaces. Surface (b) was processed with the condition $\mathrm{A}$ of $\mathrm{CO}_{2}$ jet. Surface (d) was processed with the condition $\mathrm{B}$ of $\mathrm{CO}_{2}$ jet. Area of the images is $3 \times 3$ $\mu \mathrm{m}^{2}$. 

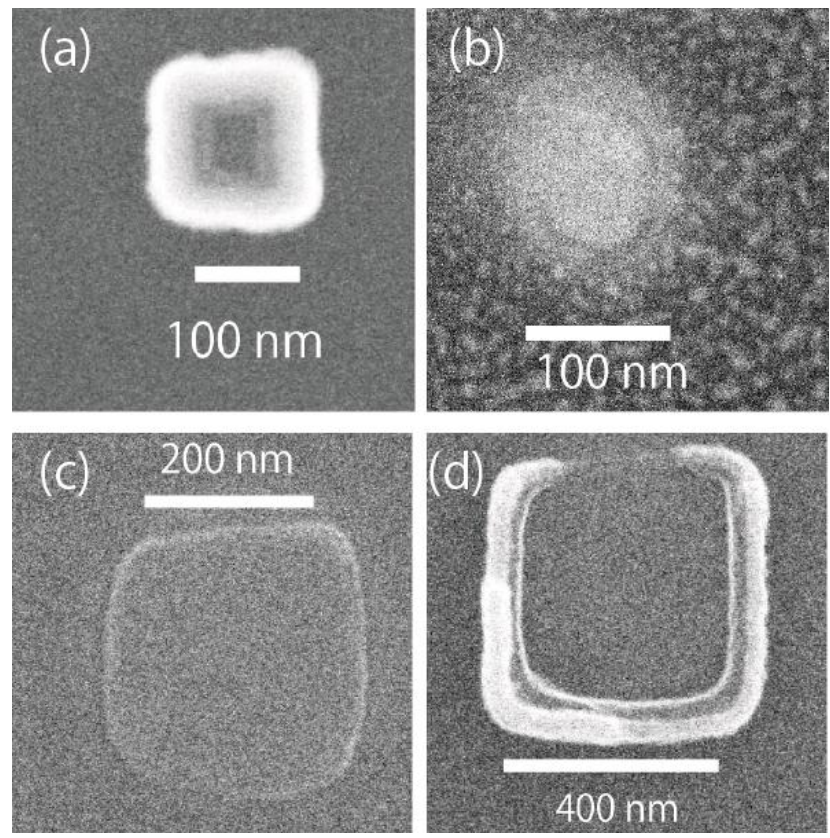

Fig. 4. Scanning electron microscopy images before and after dry ice blasting for pillar devices. (a) After coating resist mask with $\mathrm{SiO}_{2}$. (b-d) Pillars after blasting devices of different sizes.
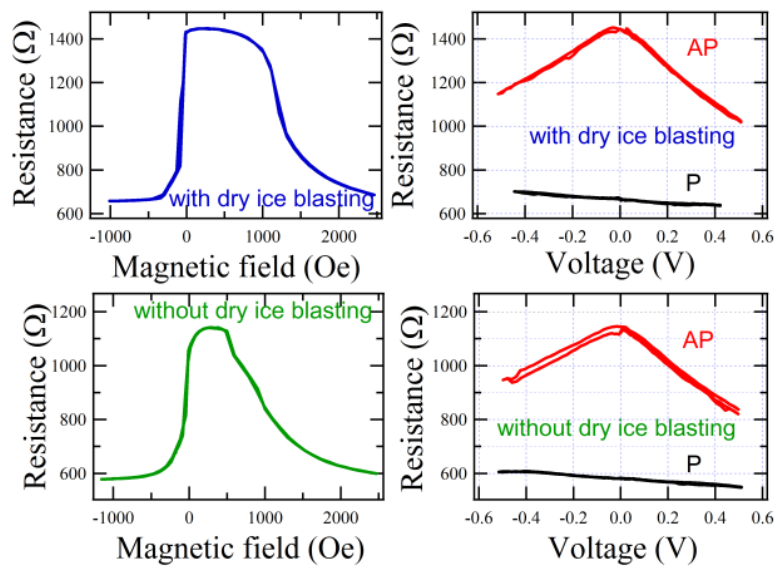

Fig. 5. Resistance versus magnetic field or electric voltage in parallel (P) and anti-parallel (AP) states for fabricated devices with or without dry ice blasting. The actual device size is estimated to be about $120 \mathrm{~nm}$ from the resistance area product.

\section{Conclusion}

Sub-micron sized pillar devices were fabricated with dry ice blasting for the lift-off process. The blasting entirely removed the resist and insulating $\mathrm{SiO}_{2}$ film, especially for smaller pillars of planar size $<250 \mathrm{~nm}$. The fabricated devices showed little difference in transport properties compared to devices made with a conventional lift-off method. A sufficiently soft and pure dry ice jet that did not cause damage to devices was achieved using a small orifice $(\phi 0.15 \mathrm{~mm})$ in the nozzle.

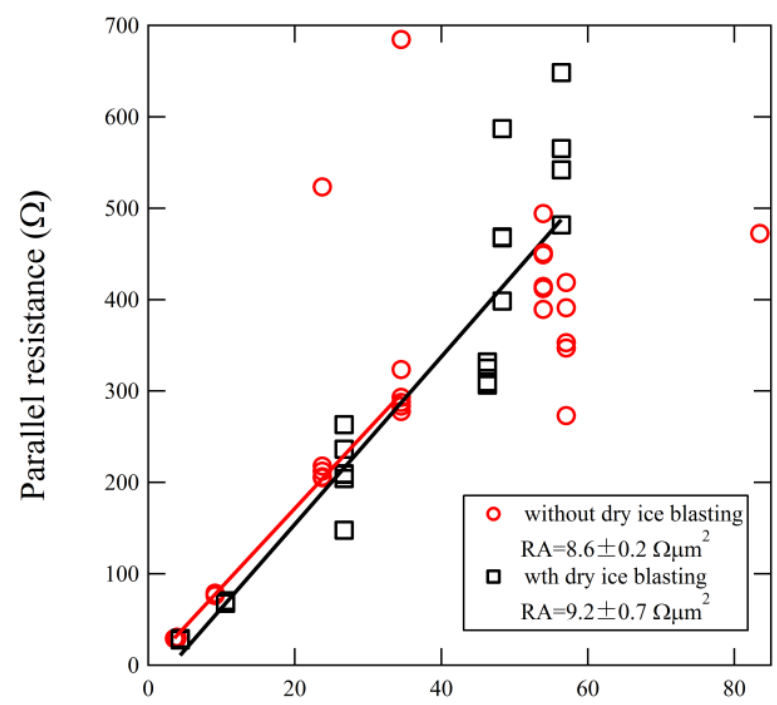

Reciplocal device area $\left(\mu \mathrm{m}^{-2}\right)$

Fig. 6. Parallel resistance versus reciprocal area plots for MTJ devices evaluated on two different wafers with (black square) and without (red circle) dry ice blasting process. The solid line indicates the linear fitting line. The resistance variation in the smaller devices is due to the lack of size reproducibility in the fabrication.

Acknowledgements This research was partly supported by METI through its "R\&D Subsidiary Program for Promotion of Academia-Industry Cooperation. This work was partly supported by JSPS KAKENHI, Grant-in-Aid for Young Scientists (B) 25870052 .

\section{References}

1) Robert Sherman, John Grob and Walter Whilock: J. Vac. Sci. Technol., B 9, 1970 (1991).

2) Robert Sherman, Drew Hirt and Ronald Vane: J. Vac. Sci. Technol., A 12, 1876 (1994)

3) Yi-Hung Liu, Hiroyuki Maruyama and Shuji Matsusaka: Advanced Power Technology, 21, 652 (2010).

4) Yi-Hung Liu, Graham Calvert, Colin Hare, Mojtaba Ghdiri and Shuji Matsusaka: Journal of Aerosol Science, 48, 1 (2012).

5) http://www.epoch-j.com/profile.htm (As of January 1, 2015)

Received Oct. 9, 2014; Accepted Mar. 4, 2015; 